
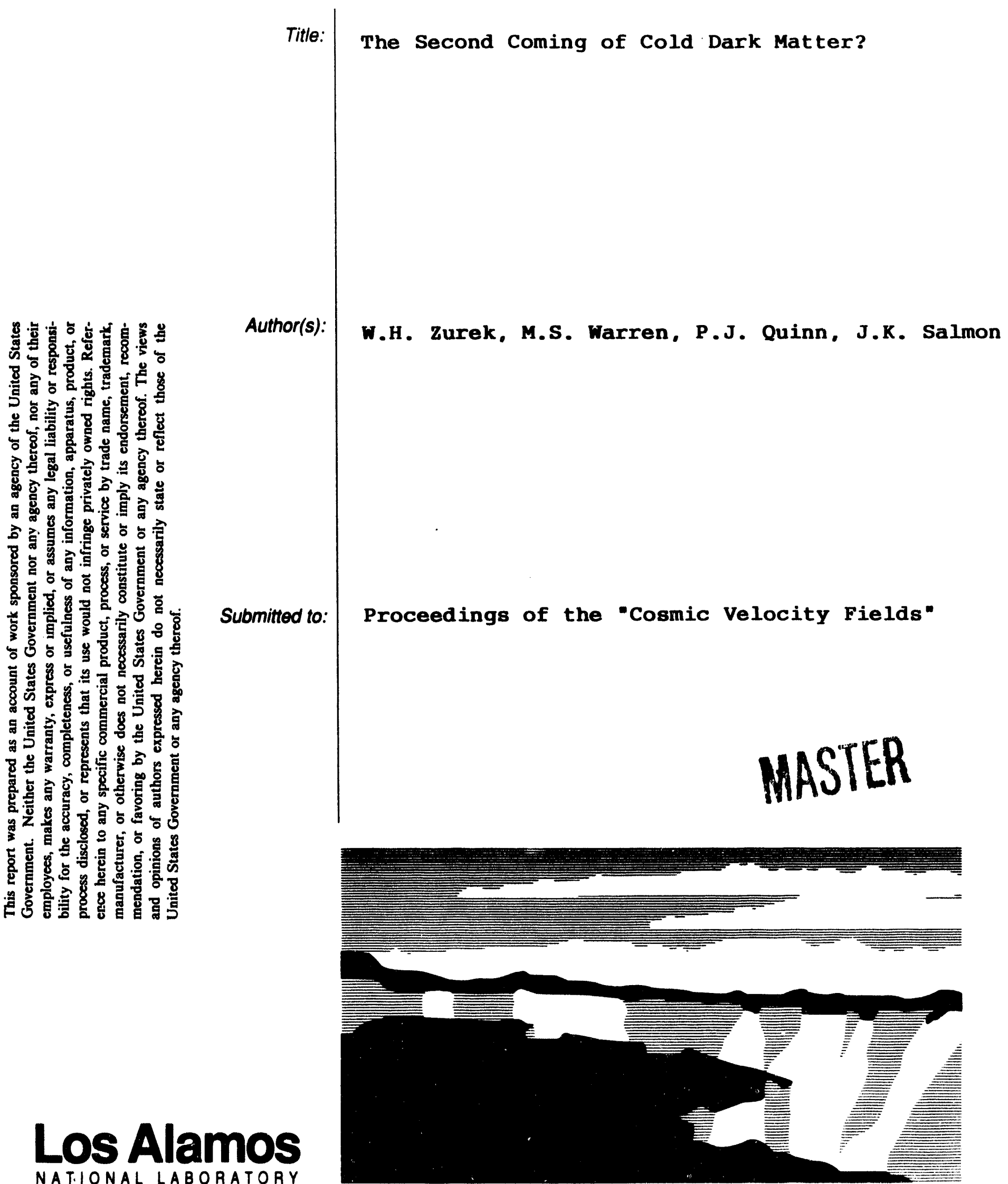

Los Alamos National Laboratory, an aftirmative action/equal opportunity empldyer, is operated by the University of California for the U.S. Department of Energy under contract W-7405-ENG.36. By acceptance of this article, the publisher recognizes that the U.S. Government retains a nonexclusive, royalty-free license to publish or reproduce the published form of this contribution, or to allow others to do so, for U.S. Government purposes. The Los Alamos National Laboratory requests that the publisher identify this article as work pertormed under the auspices of the U.S. Department of Energy. 


\title{
THE SECOND COMING OF COLD DARK MATTER?
}

\author{
W. H. Zurek ${ }^{1}$, M. S. Warren ${ }^{1}$, P. J. Quinn' ${ }^{2}$ J. K. Salmon ${ }^{3}$ \\ ${ }^{1}$ Theoretical Astrophysics, MS B288, Los Alamos National Laboratory, Los Alamos, NM 87545 \\ ${ }^{2}$ Mt. Stromlo Observatory, PB Weston Creek, ACT 2611, Canberra, Australia \\ ${ }^{3}$ Caltech Concurrent Supercomputing Facility, 206-49 Pasadena, CA 91125
}

\begin{abstract}
In recent years standard cold dark matter (CDM) theory, which enjoyed a large following throughout much of the past decade, has been abandoned by virtually all of its early supporters. The most serious argument against CDM was the incompatibility between the relatively high value of the pairwise radial velocity dispersion between galaxies, $\sigma_{v}$, inferred from numerical simulation with the much lower observational estimates. We reexamine this argument in the light of our new, high-resolution, COBE-normalized simulations and conclude that $\sigma_{v}$ is significantly overestimated in simulations which do not have sufficient resolution (i.e., which have masses of galaxies comparable to the mass of $\mathrm{N}$-body particles) and that it is also difficult to reliably estimate $\sigma_{v}$ from the observational catalogues used for this purpose. We conclude that inflationary cosmology and CDM are not - contrary to the presently prevailing prejudice - incompatible with the observations of small scale peculiar velocities, as characterized, for example, by $\sigma_{v}$.
\end{abstract}

\section{Introduction}

In the post-COBE era the freedom to normalize density perturbations in order to fit the observations has been significantly curtailed. For the CDM cosmogony, this implies that the convenient measure of density perturbations on the intermediate scales,

$$
\sigma_{8}=(\delta M / M)_{8 h-1} M p c
$$

is approximately equal to unity. This would in turn imply that the freedom to "bias" the distribution of light with respect to the distribution of mass has largely disappeared as the bias parameter $b \sim 1$.

While those implications of the COBE detection of CMB anisotropies come with a substantial uncertainty (20\% standard deviation) a significantly more pronounced bias $(b \sim 2-2.5)$ was 
initially invoked to reconcile pairwise radial velocity dispersions inferred from the observations with those predicted by the early computer simulations [1]. Thus, in the wake of the COBE measurement the prevailing prejudice (which was largely pro-CDM in the eighties, but started shifting away from CDM with the reports of significant power on large scales [8]) turned against cold dark matter [3, 13]: It seemed difficult to reconcile COBE normalization of the CDM spectrun! (which, incidentally, alleviated many of the large-scale problems) with the observational indications hat on smaller scales the real Universe appears to be simply way too peaceful.

There are several small-scale indicators of the size of mass perturbations, all of which point to a value of $\sigma_{8}$ less than unity. The most prominent among them is radial pairwise velocity dispersion $\sigma_{v}$. The usually quoted observational estimate of $\sigma_{v}$ on megaparsec scales is,

$$
\sigma_{v}^{D P}=340 \pm 70 \mathrm{~km} / \mathrm{s},
$$

and dates to a classic paper of Davis and Peebles [2] from 1983. By contrast, early simulations of CDM cosmology (with relatively few particles) yielded $\sigma_{v} \sim 1200-1500 \mathrm{~km} / \mathrm{s}$ on similar scales. This was rightly deemed unacceptable, and a bias factor $b \sim 2.5$ was introduced to bring down the simulated $\sigma_{v}$ to approximately $500 \mathrm{~km} / \mathrm{s}$, closer to the observational range [1].

A somewhat more modest bias $b \sim 1.5$ (or, equivalently, $\sigma_{8} \sim 0.7$ ) appears to be called for by the observed abundance of Abell clusters [13]: If the perturbations were normalized to $\sigma_{8} \sim 1$, there would be too many deep potential wells, which would be necussarily quite visible in X-rays (if not as optically rich clusters).

In view of these developments we have decided to simulate formation of structure in CDM Universe with a high-resolution $\mathrm{N}$-body code. Our numerical methods and some of the related astrophysical results are discussed elsewhere $[14,10,11]$. Here we shall focus on one aspect of our simulations: Pairwise radial peculiar velocities, and their comparison with observations.

\section{Simulations}

We have carried out four separate simulations with the initial conditions appropriate for CDM cosmology with $\sigma_{8} \simeq 0.65,0.7,1.05$ and 1.6 respectively. Three of these simulations dealt with a spherical fragment of CDM Universe with a diameter of $250 \mathrm{Mpc}$, while one (the one with $\sigma_{8} \sim 0.65$ ) was of a 100 Mpc diameter sphere.

As our simulations had either $N \sim 8.8 \times 10^{6}$ or $N \sim 1.76 \times 10^{7}$ particles each, halos with hundreds of virialized particles formed abundantly in all of them; by $z \approx 0$ there were typically $10^{4}$ such halos per simulation (see Figs. 1-2. for an illustration of halo and particle distributions in a typical run). High spatial resolution (20 kpc Plummer smoothing) of our models appears to have prevented massive overmerging (reported by some of the earlier simulations $[12,7]$ ). This has led to halos with rather reasonable amplitudes of rotation curves (see Fig. 3).

This does not mean that we have no overmerging: Halo-halo correlation functions (not shown here, but reproduced in [14]) follow the observed power law shape on a few Mpc scales, but are a bit more shallow on smaller sub-megaparsec scales. We regard this as evidence for a possible deficiency of objects with small ( $\sim 100 \mathrm{kpc}$ ) separations, which is most logically attributed to merging.

Correlation functions tend to be equal to unity on scales $\sim 4-5 h^{-1} \mathrm{Mpc}$ for $\sigma_{8}$ of $\sim 0.7$ and $\sim 1.06$ (i.e., somewhat less than the observed $5.4 h^{-1} \mathrm{Mpc}$ ). Moreover, on submegaparsec scales halos are significantly less correlated than mass. This slight "antibias" of halo distribution is probably also due to mergers, as the correlation between the average masses of the halos and the density of their environment indicates.

To summarize this section, we note that sizes of galactic halos, their distribution, shapes of their rotation profiles and their amplitudes, as well as the overall appearance of the CDM Universe with 


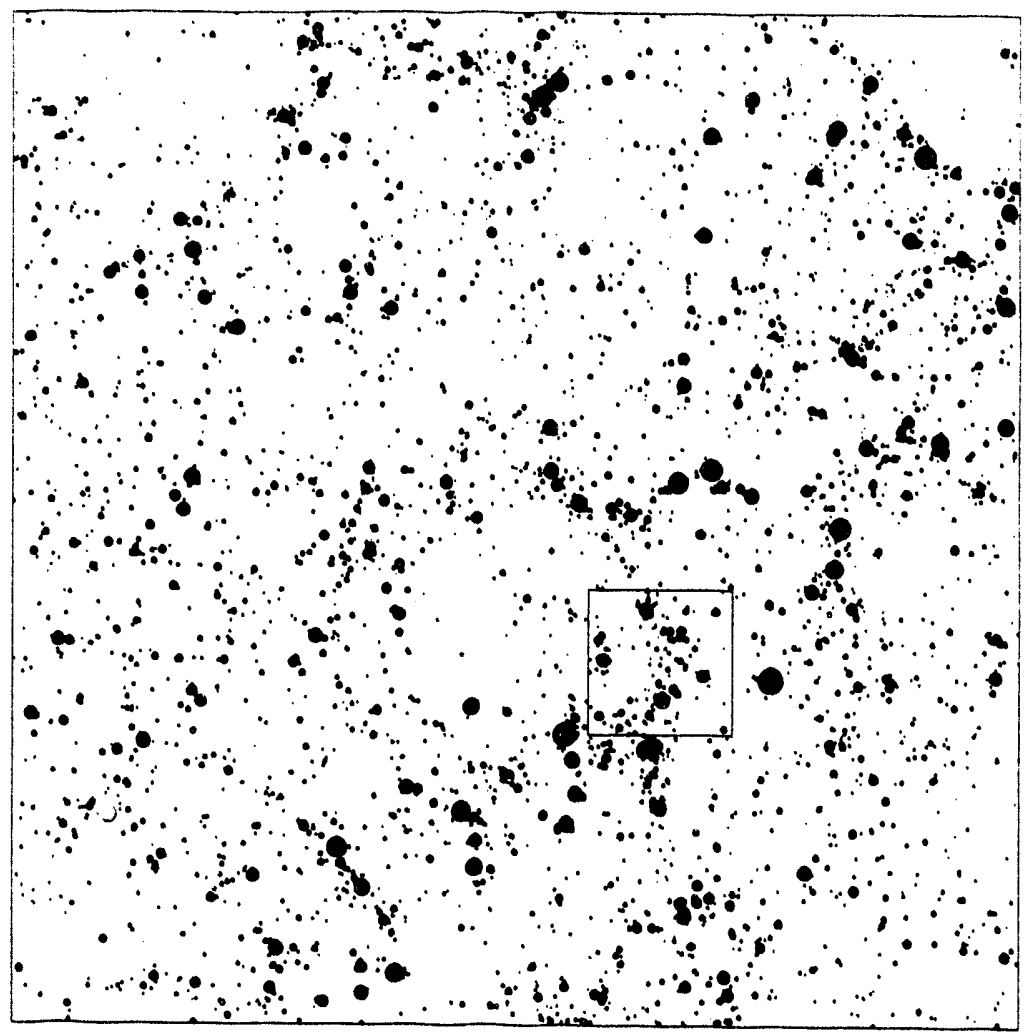

Figure 1: Distribution of halos projected onto $x-y$ plane for one of our simulations $\left(\sigma_{8} \simeq 0.7\right)$. The size of the halos are indicated by the size of the "dots". The size of the region shown is about 180 Mpc across. The small square is shown in more detail in the next figure.

normalization close to the one inferred directly for COBE $\left(\sigma_{8} \sim 1.05\right)$ or somewhat below COBE $\left(\sigma_{8} \sim 0.7\right)$ on the scales investigated in our simulations are not in any obvious contradiction with the observations.

\section{Small Scale Peculiar Velocities and Related Issues}

Cold dark matter cosmology never had much of a problem in accounting for spatial structure on small and megaparsec scales. Discrepancies between the real Universe and the distribution of dark matter could be always blamed on very real uncertainties in the physical processes involving baryons and crucial to "lighting up" of galaxies. A brief overview of our results in the last section confirms this impression: There are no glaring discrepancies in the large scale spatial structure, and the previously reported problems of overmerging appear to have been caused by poor resolution, since they seem to have largely disappeared in our higher-resolution simulations.

CDM ran into difficulties only when it had to simultaneously account for both the spatial distribution and the motions of halos. In particular, the bias parameter was invented to allow smoothing out of the underlying distribution of mass with respect to the distribution of light, so that pairwise radial velocity dispersions predicted by the numerical simulations can be brought close to the observed range.

With the normalization fixed to COBE, much of the freedom to "tune" perturbations of velocities on small scales - and, therefore, peculiar velocities - disappears. The freedom that is left is both due to the $\sim 20 \%$ standard deviation in the COBE measurement of $\delta T / T$ on the Hubble radius scale, and due to the fact that (as we shall see in the next section) inflation has an end predicts a small, but certainly nonnegligible $(\sim 30 \%-50 \%)$ correction to the power anticipated in the CDM Universe on 


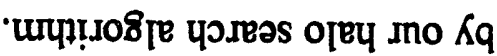

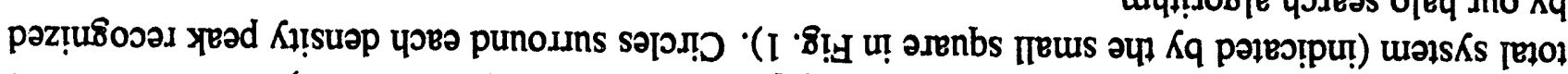

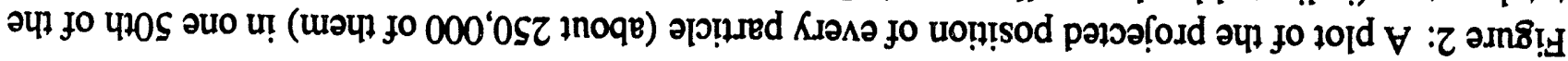

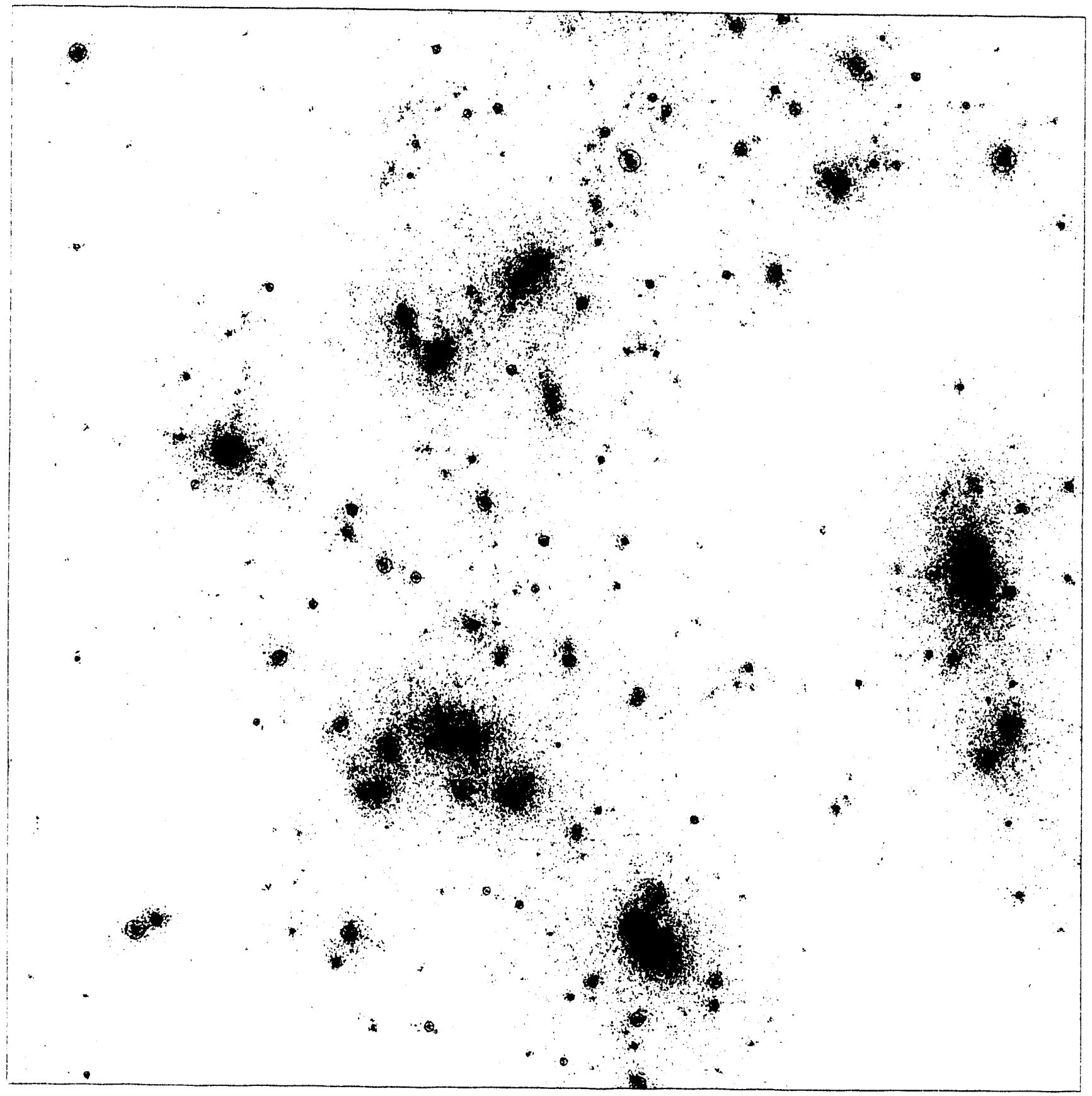




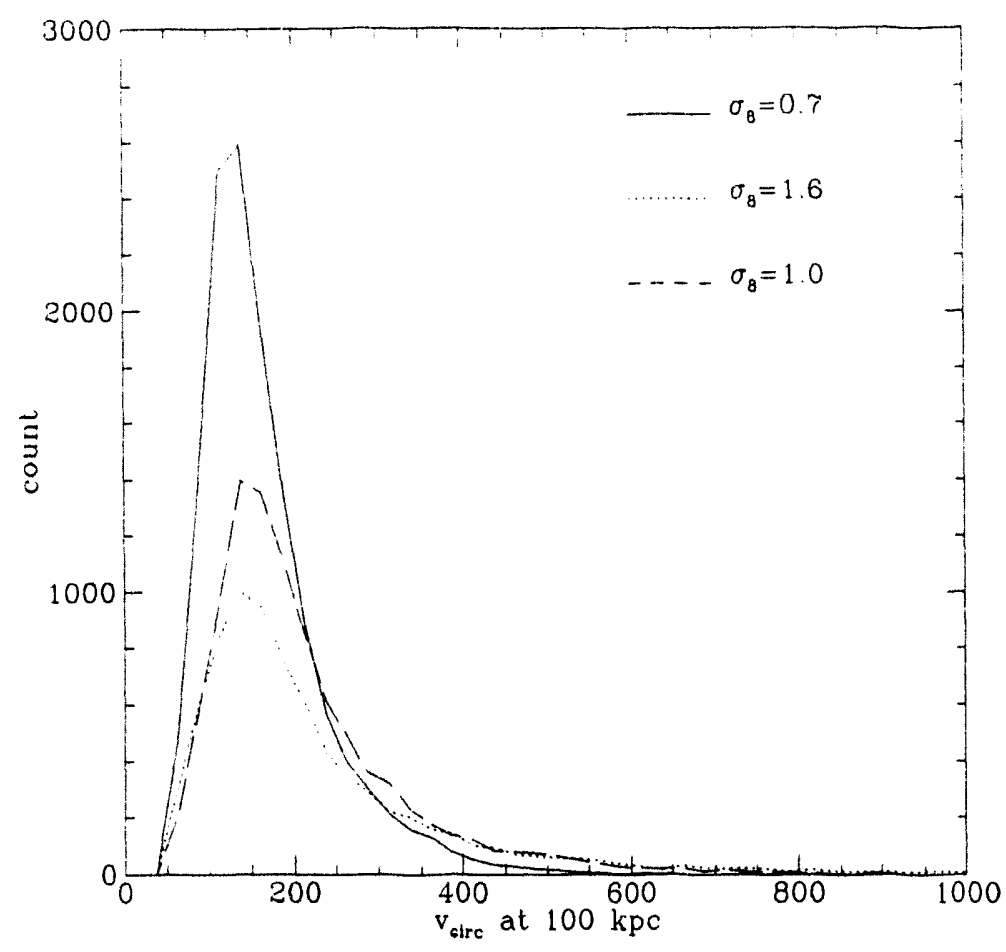

Figure 3: Distribution of the amplitudes of rotation profiles of halos formed in some of our simulations. For all of the simulations the least massive halos still resolved by the $\mathrm{N}$-body code are most abundant. Thus, the peak at $v_{\text {rot }} \sim 150 \mathrm{~km} / \mathrm{s}$ is due to the cutoff resulting from our finite resolution. On the other hand, there are very few halos with "excessive" rotation velocities, especially in the run with $\sigma_{8} \sim 0.7$, which, as we will remark in section 4 , may be the best estimate of $\sigma_{8}^{(C D M)}$ on small scales given (i) COBE normalization on the horizon scale and (ii) "generic" inflationary scenarios for the origin of perturbations.

megaparsec scales.

Peculiar velocities estimated with the full knowledge of 3-D distribution of halos from the obvious (but, as we shall shortly see, not quite correct) formula,

$$
\varsigma_{v}^{2}=\sum_{i} \sum_{j} \delta\left(v_{i, j}\right)_{r}^{2}
$$

where $\delta\left(v_{i, j}\right)_{r}$ stands for a pairwise difference of peculiar velocities between particles $i$ and $j$ are plotted in Fig. 4 for one of the undernormalized runs. On the scale of $1-2 \mathrm{Mpc}$ these velocities are typically of the order of $600-700 \mathrm{~km} / \mathrm{s}$ for halos. By contrast, particles move faster, with velocities $\sim 1000 \mathrm{~km} / \mathrm{s}$ on the same scales. Thus, there is a noticeable, although not dramatic velocity bias $b_{v} \sim 0.7$. Similar results obtain for other simulations, as $\sigma_{v}$ appears to be approximately a linear function of the normalization of the spectrum (i.e. $\sigma_{8}$ ).

The question now arises: Is the result of our COBE normalized CDM simulations compatible with the observed pairwise radio velocity dispersions on megaparsec scales (which are usually thought to fall in the range of $300-400 \mathrm{~km} / \mathrm{s}$ )? To address it, let us first note that, at least for the undernormalized runs, the value of $\sigma_{v}$ for halos is on these scales rather close to the values which were sought after by means of a much more significant bias [1]. Moreover - and, perhaps, more importantly - observational estimates of $\sigma_{v}$ are rather uncertain. Furthermore, the quantity estimated from observations is not given by Eq. (1), but, rather, is a second moment of the distribution of pairwise radial peculiar motions. The significance of the distribution between $\sigma_{v}$ and $\varsigma_{v}$ is best abpreciated 


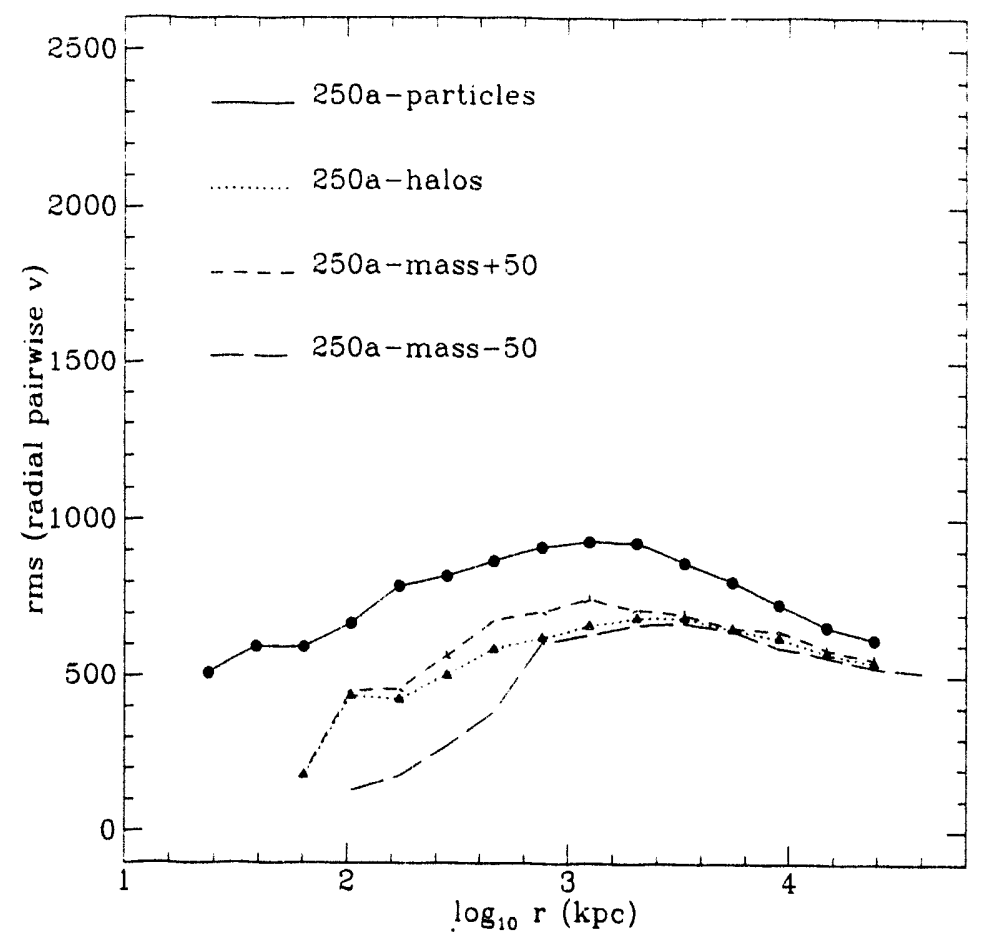

Figure 4: Root mean square pairwise radial velocity dipersion $\varsigma_{v}$ for particles and halos in a simulation with $\sigma_{8}=0.7$. Note the scale-dependence of the velocity bias. The two additional curves for halos correspond to the heavy and light halos. Heavy halos tend to form in dense neighborhoods, and therefore tend to have higher velocity dispersions.

in Fig. 5, where the distribution of velocity differences for particles separated by one megaparsec is plotted.

The first clearly noticeable fact about this distribution is that it is very well approximated by an exponential $\sim \exp \left(-\mid \delta_{v}-\langle\delta v>| / \sqrt{2} \sigma_{v}\right)$ as it is indicated by a nearly perfect triangular shape of the logarithm of the distribution:

The second important thing to note about Fig. 5 is that the "summit" of the triangle is offset from zero in the negative direction. That is, on the average, halos are falling onto one another. This is no surprise. But it does imply that the naive formula for $\varsigma_{v}$ written with the purpose of estimating $\sigma_{v}$ - calculated directly in Fig. 4 from the full $3 \mathrm{~d}$ knowledge of the co-ordinates - is inadequate as it hopelessly mixes up the first two moments.

In other words,

$$
\sigma_{v} \approx \sqrt{\varsigma_{v}^{2}-<\delta v>^{2}}
$$

where $\langle\delta v\rangle$ is the first moment - the average radial infall - and is shown in Fig. 6. Let us hasten to add that $\langle\delta v\rangle$ is typically smaller than $\varsigma_{v}$ by a factor of 2-3. Thus, in our simulations (as well as, presumably, in the real Universe) the difference between $\sigma_{v}$ and $\varsigma_{v}$ is the order of $20 \%$. Therefore, while this is undoubtedly a correction which will bring the numerical predictions for $\varsigma_{v}$ into the range of $500-600 \mathrm{~km} / \mathrm{s}$, that is, closer to the observational estimates, it would not be sufficient to decisively alter the conclusion of our study.

It is perhaps more important to note that the observational estimates of $\sigma_{v}$ are obtained in a still different manner which must - of necessity - use only redshifts and angular positions of the galaxies to infer enough about their three-dimensional distribution and their velocities to eventually extract $\sigma_{v}$. We shall describe the procedure (discussed in detail by Davis and Peebles) only very 


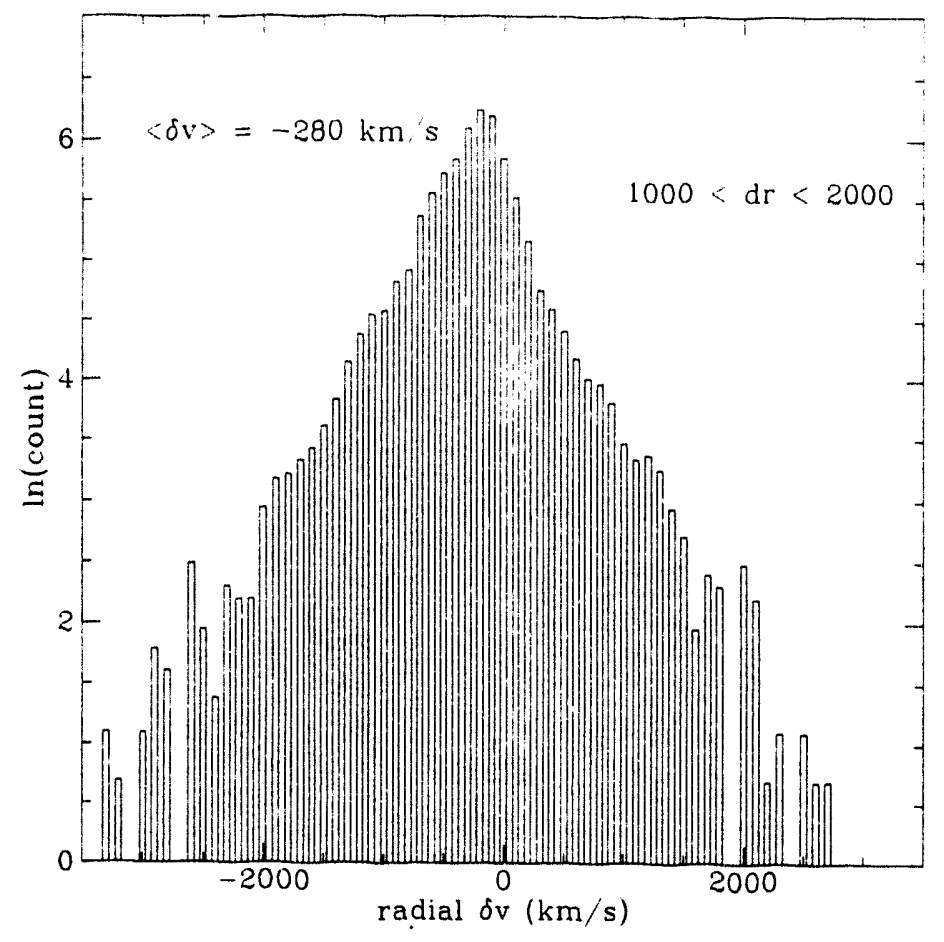

Figure 5: Distribution of pairwise radial velocity for pairs of halos separated by 1-2 Mpc. The triangular shape of this distribution over the large range of $\delta v$ implies that the distribution of pairwise velocities is indeed exponential, and that its second moment $\sigma_{v}$ can be inferred from the "slope" of the triangle, after the first moment (which offsets the center of the triangle towards negative $\delta v$ by $\langle v\rangle=280 \mathrm{~km} / \mathrm{s}$ in the case illustrated here) is properly taken into account.

briefly: In essence, one compares the inferred galaxy-galaxy correlation function in the direction transverse to the line of sight (where it is, to the first order, unaffected by the peculiar velocities) with the measured correlation function in redshift. In absence of peculiar velocities the two correlation functions would coincide. Peculiar velocities "wash out" correlations in redshift thus making the corresponding correlation function less steep. One can infer $\sigma_{v}$ from the new, shallower slope. Thus, one is using the phenomenon responsible for "fingers of God" in clusters to estimate $\sigma_{v}$.

It should be pointed out that an average infall (measured by the first moment of the velocity distribution $\langle\delta v\rangle$ ) has the opposite effect: Galaxies move towards each other. Thus, providing that $\langle\delta v>$ is smaller than the Hubble flow on the considered scales, the galaxies moving together will appear to be closer - the redshift correlation function will be steepened compared to the transverse, "true spatial" correlation function - as a result of net infall $\langle\delta v\rangle$.

Clearly, as $\sigma_{v}$ and $\langle\delta v>$ "work in the opposite directions," one must assume something about one of them in order to infer the other. Such assumptions about $\langle\delta v>$ have been put forward by Davis and Peebles [2] — and we have, in effect, followed their procedure (including background analysis) to extract $\sigma_{v}$ from our simulations in a manner analogous to the observational efforts.

The key conclusion of our calculations is that when the sample is large enough (i.e. a whole simulation with $\sim 10^{4}$ halos) Davis and Peebles (DP) procedure yields estimates of $\sigma_{v}$ which are typically within $\sim 10-20 \%$ of the values derived with the full three dimensional knowledge of the locations and velocities of galaxies. Unfortunately, observational samples are usually smaller. For example, the northern sky CfA sample contains only $\sim 1800$ galaxies, $\sim 1200$ of which are left when the DP magnitude selection criteria are imposed.

To see how DP procedure works in such circumstances, we have cut out from our simulation 

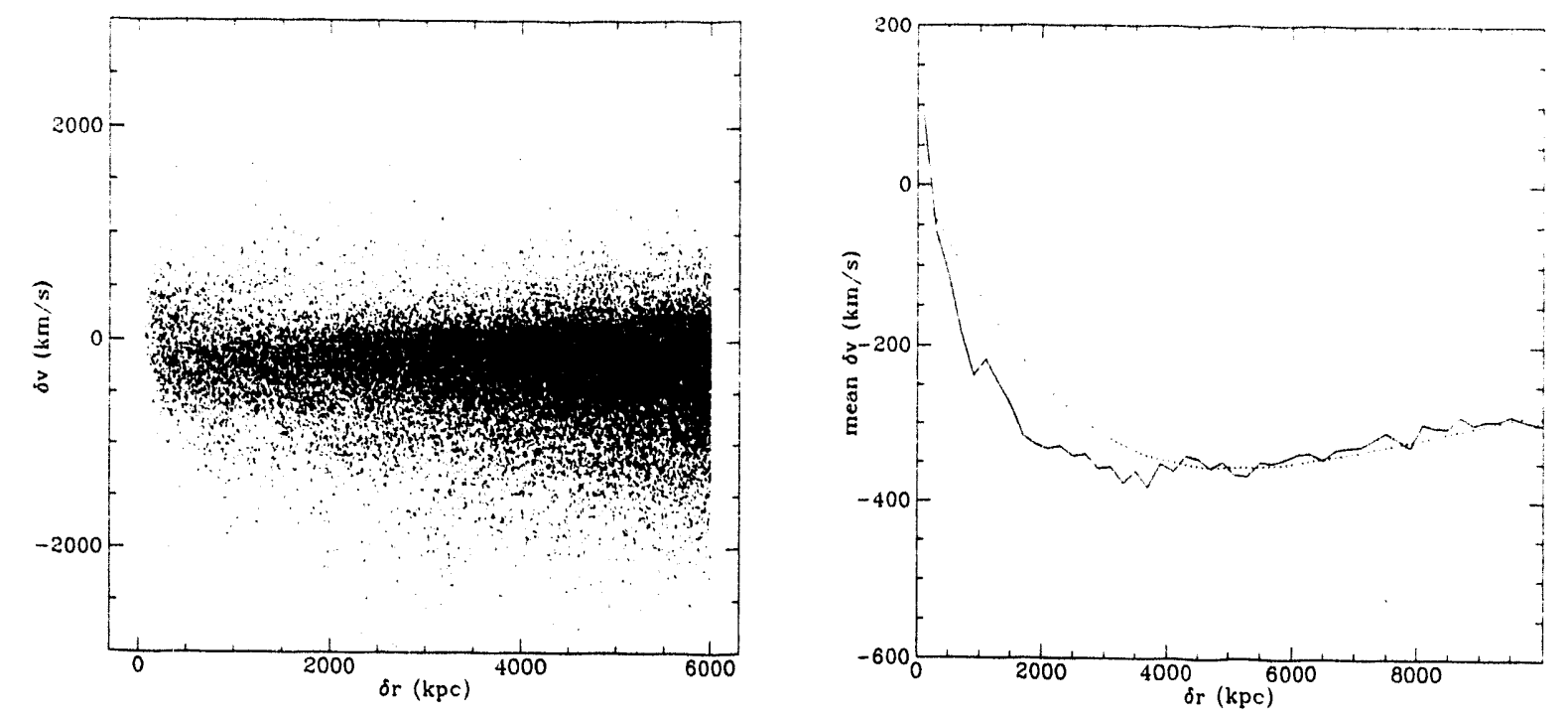

Figure 6: a.) Both the overall infall and the velocity dispersion are shown in this figure as a function of the separation between halos. Note that the pairwise velocities tend to be more often negative than positive, which results in an overall infall. b.) The average value of the infall - the first moment of the distribution shown in Fig. 6(a) - is plotted as a function of the separation between halos. On large scales the infall model proposed by Davis and Peebles (dotted line) seems to be a reasonable fit, but it does not yield a very convincing fit on small ( $\sim 1 \mathrm{Mpc})$ scales.

samples with similar "CfA" geometry and comparable numbers of halos. With these, much smaller, samples, the DP procedure was no longer as successful as for the whole simulation. For example, the inferred $\sigma_{v}$ ranged between 450 and $850 \mathrm{~km} / \mathrm{s}$ for eight different "CfA-like" samples cut out from the san., model for which fully 3-D analysis yielded $\sigma_{v} \sim 600 \mathrm{~km} / \mathrm{s}$.

This large scatter of the inferred values of $\sigma_{v}$ is not too surprising when one realizes that the analysis is dominated by a few dense structures. This is because the number of pairs is (approximately) proportional to the square of the spatial density of galaxies. Hence, clusters (which also happen to have highest $\sigma_{v}$ ) are bound to significantly influence the value of $\sigma_{v}$ averaged over the whole sample.

A good illustration of this fact is afforded by our reanalysis of the CfA northern sky catalogues. Again, we have followed the DP procedure, although, in the application to this particular sample there are a few additional issues which must be addressed (and which underscore the difficulty of faithfully repeating the "observational" procedure in the context of the numerical simulation).

Let us begin by saying that when we have naively followed the same DP procedure we have previously successfully used to get "observational" estimates of $\sigma_{v}$ from our simulations, we have obtained $\sim 600 \mathrm{~km} / \mathrm{s}$ for CfA northern sky catalogue. Two important aspects of this analysis had to be refined to arrive at the lower (and usually quoted) values of $\sigma_{v}$ : (i) The sample selection. (ii) Trtatment of the immediate vicinity of the core of the Virgo cluster.

Davis and Peebles corrected their estimates of the absolute magnitudes of the CfA galaxies by taking into account the overall Virgocentric infall in converting redshifts into distances. This step seems to be innocent, but is in fact fairly crucial, as without it one obtains a sample with less than 1000 galaxies. When this correction is introduced, and the apparent and absolute magnitude criteria are reapplied to the whole 1800 CfA galaxies, a "richer" sample of $\sim 1200$ galaxies is obtained. The "new" $\sigma_{v}$ computed for this sample is somewhat smaller $(\sim 450-500 \mathrm{~km} / \mathrm{s}$, with its value dependent on the exact manner in which the correction for infall is implemented) but it still seems to be larger than the $\sigma_{v}^{D P}=340 \mathrm{~km} / \mathrm{s}$ inferred by Davis and Peebles.

We have found it impossible to get $\sigma_{v}^{D P}$ without one additional correction: We had to either get rid 
of the $\sim 50$ galaxies in the core of the Virgo cluster, or subtract a specific model of the Virgocentric motion in the triple infall region of Virgo. Only with these "surgical" steps we were able to bring down our estimates of $\sigma_{v}$ from the CfA survey below $340 \mathrm{~km} / \mathrm{s}$ (Mo et al., 1993, ([9]) have reached similar conclusions on this issue.)

In our view, implications of these considerations for the CDM cosmogony are quite dramatic. The pairwise radial velocity dispersion, $\sigma_{v}$, is no longer a stringent constraint. This is both due to the intrinsic (random) errors which cause large variations of the inferred $\sigma_{v}$ for the CfA-like samples, and due to the fact that when exactly the same "naive" DP procedure is followed for both our simulations and for the CfA sample (i.e. when no Virgo-related corrections are made), similar values of $\sigma_{v} \sim 600$ $\mathrm{km} / \mathrm{s}$ are inferred, at least for the somewhat undernormalized $\left(\sigma_{8} \sim 0.7\right)$ models. (We shall see in the next section that in the COBE-normalized CDM model inflation actually favors small scale $\sigma_{8}$ close to 0.7 .)

Broader implications of our results are also important. The first of them: $\sigma_{v}$ is not a very robust diagnostic of the dynamics of the system. This is because - in contrast to the other statistical ensembles, such as an equilibrated gas where temperature is in effect an equivalent of $\sigma_{v}$ - peculiar velocity dispersion is not an easily defined property of the system as a whole. Rather, it can differ by an order of magnitude between the dense clusters and "local backwater" like our local group. As the averaging procedure is quite sensitive to the local density (the number of pairs is proportional to the square of density) and as the contributions of the pairs are added with the squares of individual pairwise peculiar velocity differences, clusters can easily gain an overwhelming influence on the inferred average $\sigma_{v}$. By the same token, samples which "stay away" from the clusters (i.e. the IRAS sample [6]) can significantly underestimate $\sigma_{v}$. Moreover, observational methods of estimating $\sigma_{v}$ and $\langle\delta v\rangle$ inextricably mix these two characteristics of the velocity field, further complicating the inference process.

A corollary of these remarks is that the value of $\sigma_{v}$ is going to be very sensitive to the sample selection process: Samples which "avoid" cluster cores will yield relatively small $\sigma_{v}$. Samples which contain rich clusters are likely to have the estimated value of $\sigma_{v}$ overwhelmed by the velocity dispersion in the cores. (This seems to be already the case for the CfA sample, as our discussion above demonstrates.) Consequently, samples such as IRAS (which select only late-type galaxies) are bound to underestimate the value of $\sigma_{v}$. A more careful discussion of these effects (as well as of their implications for the estimates of $\langle\delta v\rangle$ ) will be given elsewhere.

\section{$4 \mathrm{CDM}, \mathrm{COBE}$, Inflation, and the Value of $\sigma_{8}$}

It is a common perception that: (1) Inflation implies an exact Harrison-Zeldovich spectrum for the primordial density perturbations, $P(k) \sim k$. (2) COBE anisotropies are only due to density perturbations. If these two statements were exactly valid, $\sigma_{8}$ inferred from the anisotropies of the microwave background would be somewhat larger than unity $\left(\sigma_{8} \sim 1.06\right)$ with an approximately $20 \%$ standard deviation.

Common perception summarized above is only approximately correct since ([4]): (i) The very fact that inflationary epoch has an end necessarily implies that the exponent of the primordial power law is somewhat smaller than unity (i.e. $P(k) \sim k^{1-\epsilon}$, where $\epsilon \sim 0.03-0.05$. (ii) In addition to scalar [S] (density) perturbations COBE signal is due to the tensor [T] (gravity wave) contribution which can account for a significant fraction of the observed temperature variations $T / S, \simeq 7 \epsilon$.

Both of these effects taken together imply that the density perturbations - the seeds of the collapsing structures - will have a significantly smaller amplitude with the typical $\sigma_{8}$ in the range $0.65-0.8$. Thus our "undernormalized" simulations are actually closer to the value of $\sigma_{8}$ relevant for the small scales. 
This bodes well for the chances of CDM not just because of the smaller values of $\sigma_{8}$ : As it was recently pointed out in [13], $\sigma_{8} \sim 1$ are incompatible with the observed abundances of Abell clusters, which form as a result of perturbations on scales close to $\sim 8 h^{-1} \mathrm{Mpc}$ (hence, change of spectral shape cannot help!). The value of $\sigma_{8}$ close to $\sim 0.7$ is much more comfortable as it can be most likely reconciled both with the cluster abundance data, and might be still sufficiently large to account for the large-scale flows, as implied by POTENT [5].

\section{References}

[1] M. Davis, G. Efstathiou, C. S. Frenk, and S. D. M. White. The evolution of large-scale structure in a universe dominated by cold dark matter. Ap. J., 292:371, 1985.

[2] M. Davis and P. J. E. Peebles. Ap. J., 267:465, 1983.

[3] M. Davis, F. J. Summers, and D. Schlegel. Nature, 359:393-396, 1992.

[4] R. L. Davis, H. M. Hodges, G. F. Smoot, P. J. Steinhart, and M. S. Turner. Phys. Rev. Let., 69:1856-1859, 1992.

[5] A. Dekel, E. Bertschinger, and S. M. Faber. Ap. J., 364:370-395, 1990.

[6] K. B. Fisher, M. Davis, M. A. Strauss, A. Yahil, and J. Huchra. M. N. R. A. S., 1993. (submitted).

[7] J. M. Gelb, B. Gradwohl, and J. A. Frieman. Ap. J. (Letters), 403:5, 1993.

[8] S. J. Maddox, G. Efstathiou, W. J. Sutherland, and J. Loveday. M. N. R. A. S., 242:43P, 1990.

[9] H. J. Mo, Y. P. Jing, and G. Bömer. M. N. R. A. S., 1993. (in press).

[10] M. S. Warren and J. K. Salmon. A parallel hashed oct-tree N-body algorithm. preprint LAUR93-1224, LANL, 1993.

[11] Michael S. Warren and John K. Salmon. Astrophysical N-body simulations using hierarchical tree data structures. In Supercomputing '92. IEEE Comp. Soc., 1992.

[12] S. D. M. White, M. Davis, G. Efstathiou, and C. S. Frenk. Nature, 330:451, 1987.

[13] S. D. M. White, G. Efstathiou, and C. S. Frenk. M. N. R. A. S., 262:1023-1028, 1993.

[14] W. H. Zurek, P. J. Quinn, J. K. Salmon, and M. S. Warren. Large scale structure after COBE: Peculiar velocities and correlations of cold dark matter halos. Ap. J., 1993. (submitted). 

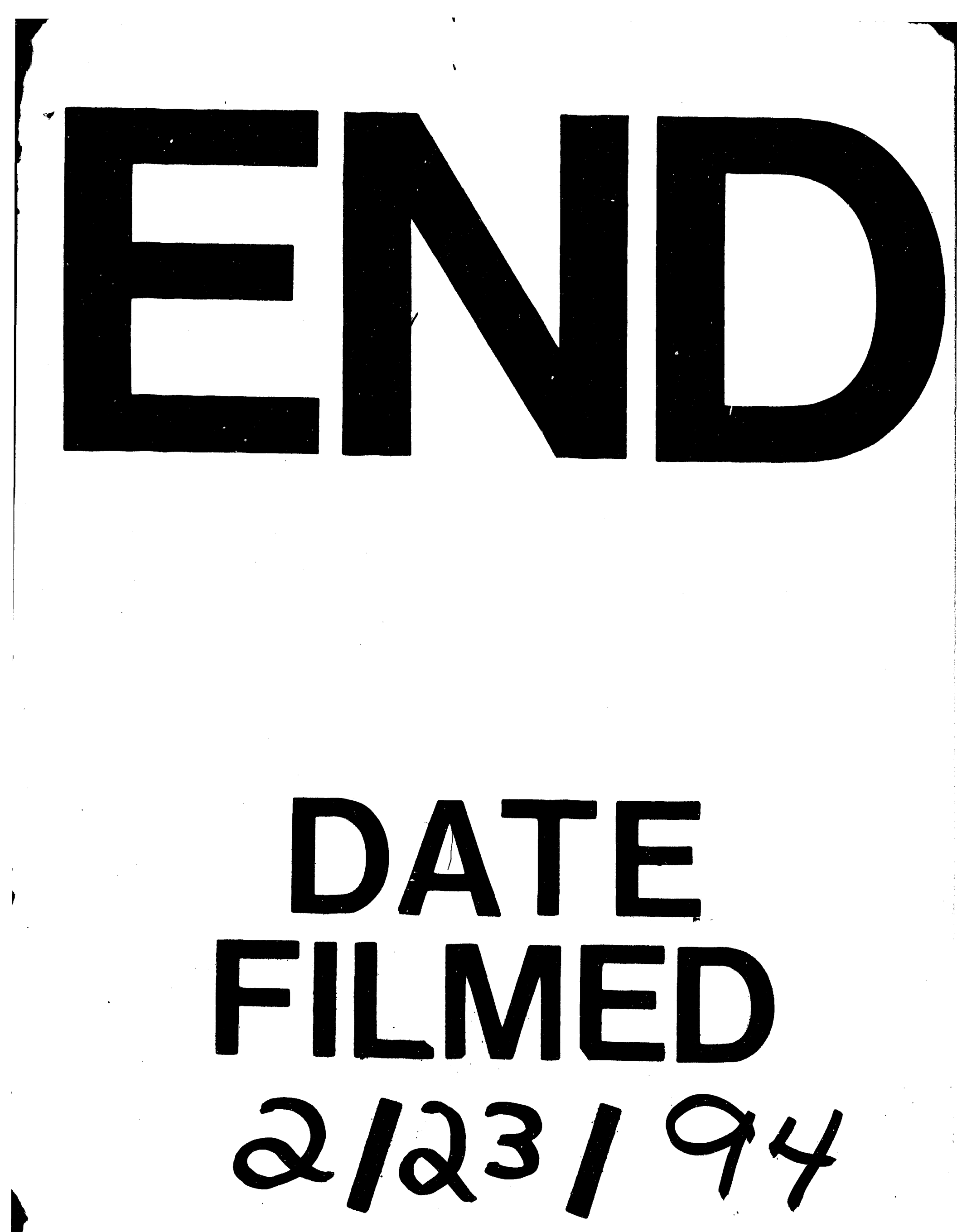

$\mid$ 